\title{
Focus on immune-related adverse events (irAEs) in immunotherapy of hepatobiliary malignancies
}

\author{
Lei Zhang, Haitao Zhao
}

Department of Liver Surgery, Chinese Academy of Medical Sciences and Peking Union Medical College (CAMS \& PUMC), Peking Union Medical College Hospital, Beijing 100032, China

Correspondence to: Haitao Zhao. Department of Liver Surgery, Chinese Academy of Medical Sciences and Peking Union Medical College (CAMS \& PUMC), Peking Union Medical College Hospital, Beijing 100032, China. Email: zhaoht@pumch.cn.

Provenance and Peer Review: This article was commissioned by the editorial office, Hepatobiliary Surgery and Nutrition. The article did not undergo external peer review.

Submitted Oct 13, 2019. Accepted for publication Oct 24, 2019.

doi: 10.21037/hbsn.2019.10.25

View this article at: http://dx.doi.org/10.21037/hbsn.2019.10.25

There has been a chronic lack of effective treatment for hepatobiliary malignancies, and overall survival of comprehensive therapy, including targeted chemotherapy, is still low $(1,2)$. A series of immune checkpoint blockade drugs have offered new choices for patients with advanced hepatobiliary malignancies. Although the efficacy of a $\mathrm{PD}-1 / \mathrm{PD}-\mathrm{L} 1$ blockade is not satisfactory, comprehensive therapy combining traditional chemotherapy, targeted therapy, local treatment, and radiotherapy often have satisfactory results (3). The drugs including nivolumab, pembrolizumab, have been approved successively for hepatocellular carcinoma (4,5), satisfactory objective response rate (ORR), and disease control rate (DCR) were obtained with tyrosine kinase inhibitor (TKI) drugs (6). Therefore, immunotherapy is becoming a key part of the treatment for advanced hepatobiliary tumors.

Immune checkpoint inhibitors block the PD-1 and PDL1 pathway, which activates the function of the local $\mathrm{T}$ cells while increasing the effects of killing tumors. However, at the same time, non-tissue specific binding may result in abnormal activation of $\mathrm{T}$ cells in normal tissues with immune-related side effects. Now immune-related adverse effects known to affect all body systems, include endocrine systems (such as hypophysitis, hypothyroidism, pancreatitis, etc.), colitis, and rash, while rarely include autoimmune pneumonitis, myocarditis, etc. Some severe patients can lead to death, such as the death rate of myocarditis is about $50 \%$ (7-9). Due to the lack of experience previously, doctors are often unable to find immune-related side effects early, and many patients may delay diagnosis and treatment.

Now, immune-related adverse events (irAEs) are mainly applied to the irAE classification system for evaluation. Grade 1-2 of adverse reactions need symptomatic management and low dose hormonotherapy, while grades 3 and 4 need to stop using PD-1/PD-L1 blockade if it necessarily requires steroid pulse therapy. However, among a few patients with myocarditis and autoimmune pneumonitis, the use of TNF- $\alpha$ and IL- 6 monoclonal antibodies have been successfully controlled in a few case reports. At the same time, a large number of patients participate in the combination treatments at present, which puts forward higher requirements for doctors to distinguish the adverse effects of the treatment of PD-1 and targeted agents. As the adverse effects related to immunotherapy involve multiple disciplines, it is more important to emphasize the multidisciplinary collaboration treatment, including oncology, endocrinology, immunology, respiratory medicine, cardiology, and emergency department (8).

Although immune-related side effects may bring a series of problems during immunotherapy, the occurrence of side effects is also associated with good therapeutic responses in some patients. Recently, several studies have shown that irAEs are associated with better therapeutic effects when PD-1 antibody is used in patients with melanoma and nonsmall cell lung cancer (NSCLC) and is particularly the presence of thyroid, and other endocrine-related irAE tends to predict the better therapeutic efficacy of immunotherapy. Autoantibodies may play an important role in the emergence of irAEs and clinical benefits. The study suggests that preexisting autoantibodies may be associated with the development of irAEs and clinical benefits. The presence of autoantibodies is a marker of therapeutic efficacy during 
treatment, particularly anti-thyroid antibodies $(10,11)$.

In the future, several questions need to be clarified about the adverse effects of immunotherapy: (I) currently, few studies research on the characteristics of local immune status changes in organs involved irAEs. The relationship of changes between the local immune environment in normal tissues and the immune microenvironment in tumors remains unclear. (II) There are still no effective means to predict the time and severity of adverse reactions in immunotherapy. Early biomarker screening is of great significance in predicting the occurrence of grade 3-4 irAE and intervention at an early stage. (III) Now organs have certain specificity, which affected by adverse events of different PD-1/PD-L1 agents in the treatment of different tumor species, and the mechanism is not completely clear. So, understanding the time and space distribution of side effects of different drugs has guiding significance for drug selection and adjustment. (IV) Due to the unique mechanism of immunotherapy, about $10 \%$ of patients may appear pseudoprogression, however, to determine whether progress needs to wait for re-evaluation. Currently, there is no effective tool to distinguish the differences between hyperprogression for the first time. The appearance of irAE and the dynamic changes of the autoantibodies could be potential monitoring indicators.

\section{Acknowledgments}

Funding: None.

\section{Footnote}

Conflicts of Interest: $\mathrm{HZ}$ serves as an unpaid editorial board member of Hepatobiliary Surgery and Nutrition. The other author has no conflicts of interest to declare.

Ethical Statement: The authors are accountable for all aspects of the work in ensuring that questions related to the accuracy or integrity of any part of the work are appropriately investigated and resolved.

Open Access Statement: This is an Open Access article distributed in accordance with the Creative Commons Attribution-NonCommercial-NoDerivs 4.0 International License (CC BY-NC-ND 4.0), which permits the noncommercial replication and distribution of the article with the strict proviso that no changes or edits are made and the original work is properly cited (including links to both the formal publication through the relevant DOI and the license). See: https://creativecommons.org/licenses/by-nc-nd/4.0/.

\section{References}

1. Biselli M, Garuti F, Neri A. Hepatocellular carcinoma surveillance: an open question. Hepatobiliary Surg Nutr 2019;8:431-2.

2. Raoof M, Singh G. Rising trends in intrahepatic cholangiocarcinoma incidence and mortality: getting at the root cause. Hepatobiliary Surg Nutr 2019;8:301-3.

3. Long J, Lin J, Wang A, et al. PD-1/PD-L blockade in gastrointestinal cancers: lessons learned and the road toward precision immunotherapy. J Hematol Oncol 2017;10:146.

4. El-Khoueiry AB, Sangro B, Yau T, et al. Nivolumab in patients with advanced hepatocellular carcinoma (CheckMate 040): an open-label, non-comparative, phase 1/2 dose escalation and expansion trial. Lancet 2017;389:2492-502.

5. Zhu AX, Finn RS, Edeline J, et al. Pembrolizumab in patients with advanced hepatocellular carcinoma previously treated with sorafenib (KEYNOTE-224): a non-randomised, open-label phase 2 trial. Lancet Oncol 2018;19:940-52.

6. Kudo M. Targeted and immune therapies for hepatocellular carcinoma: Predictions for 2019 and beyond. World J Gastroenterol 2019;25:789-807.

7. Pauken KE, Dougan M, Rose NR, et al. Adverse events following cancer immunotherapy: obstacles and opportunities. Trends Immunol 2019;40:511-23.

8. Postow MA, Sidlow R, Hellmann MD. Immune-related adverse events associated with immune checkpoint blockade. N Engl J Med 2018;378:158-68.

9. Haratani K, Hayashi H, Chiba Y, et al. Association of immune-related adverse events with nivolumab efficacy in non-small-cell lung cancer. JAMA Oncol 2018;4:374-8.

10. de Moel EC, Rozeman EA, Kapiteijn EH, et al. Autoantibody development under treatment with immunecheckpoint inhibitors. Cancer Immunol Res 2019;7:6-11.

11. Ma W, Gilligan BM, Yuan J, et al. Current status and perspectives in translational biomarker research for PD-1/ PD-L1 immune checkpoint blockade therapy. J Hematol Oncol 2016;9:47.

Cite this article as: Zhang L, Zhao H. Focus on immunerelated adverse events (irAEs) in immunotherapy of hepatobiliary malignancies. HepatoBiliary Surg Nutr 2020;9(3):348-349. doi: 10.21037/hbsn.2019.10.25 